Relmecs, diciembre 2018 - mayo 2019, vol. 8, no. 2, e042, ISSN 1853-7863

Universidad Nacional de La Plata. Facultad de Humanidades y Ciencias de la Educación.

Centro Interdisciplinario de Metodología de las Ciencias Sociales.

Red Latinoamericana de Metodología de las Ciencias Sociales

\title{
Discusiones sobre la naturaleza de la investigación en psicoanálisis
}

\section{Discussions on the nature of research in psychoanalysis}

\author{
Maximiliano Azcona \\ Facultad de Psicologia,Universidad Nacional de La Plata, Argentina \\ m.azcona@psico.unlp.edu.ar
}

\section{Resumen:}

Este artículo reseña las principales perspectivas de investigación en el campo psicoanalítico y pretende dar apoyo a las siguientes conjeturas: (I) la apelación a ciertas críticas epistemológicas y metodológicas ha sido un factor fundamental para justificar el desarrollo de investigación extraclínica en el interior del campo psicoanalítico, y (II) la desestimación de la metodología del estudio de casos se sostiene en algunos presupuestos que ameritan ser revisados y discutidos.

Se concluye la necesidad de amplificar este tipo de discusiones metateóricas sobre los modos de producción de conocimiento en psicoanálisis.

Palabras clave: Psicoanálisis, Metodología , Epistemología.

\section{ABstract:}

This article review the main perspectives of research in the psychoanalytic field and aims to give support to the following conjectures: (I) the appeal to some epistemological and methodological criticisms has been a key factor to justify the development of extra-clinical research in the interior of the psychoanalytic field, and (II) the dismissal of the case study methodology is held under some budgets which deserve to be reviewed and discussed.

It is concluded the need to amplify this type of meta-theoretical discussions on the modes of production of knowledge in psychoanalysis.

KEYwORDs: Psychoanalysis, Methodology, Epistemology.

Para el psicoanalista, en las circunstancias hoy reinantes, hay una tendencia afectiva peligrosísima: la ambición de obtener, con su nuevo y tan atacado instrumento, un logro convincente para los demás (Freud, 1912/2004, p. 114).

Este artículo pretende reseñar las principales perspectivas de investigación en el campo psicoanalítico y dar apoyo a las siguientes conjeturas: (I) la apelación a ciertas críticas epistemológicas y metodológicas ha sido un factor fundamental para justificar el desarrollo de investigación extraclínica en el interior del campo psicoanalítico, y (II) la desestimación de la metodología del estudio de casos (EC de aquí en más) se sostiene en algunos presupuestos que ameritan ser revisados y discutidos.

Para ello, primero intentaré sistematizar algunas taxonomías posibles para clasificar las formas de investigar en psicoanálisis; luego expondré la apelación que un sector del psicoanálisis ha venido realizando a ciertas críticas epistemológicas sobre la insuficiencia del estudio de casos tradicional, para proponer a partir de allí la necesidad de importar o desarrollar otras metodologías de investigación. Este apoyo en las objeciones epistemológicas ha venido a empalmarse con afanes naturalistas y con demandas sociales de legitimidad, dando lugar a los estudios experimentales y las investigaciones sobre la eficacia terapéutica. Luego mostraré que estas derivaciones no han ocurrido sin resistencias en el interior de las comunidades psicoanalíticas, y que persiste una firme defensa del EC como metodología principal para la investigación en psicoanálisis. Finalmente, revisaré tres presupuestos sobre la naturaleza de los EC y abogaré por una mayor discusión crítica de los problemas metodológicos que la investigación psicoanalítica merece. 


\section{TIPOS DE INVESTIGACIÓN EN PSICOANÁLISIS}

El entramado de controversias que se desarrollan desde el siglo pasado sobre la identidad disciplinar del psicoanálisis (intentando precisar si es una ciencia, una hermenéutica o una disciplina sui generis) pareciera haber variado su punto de foco: el principal debate en la actualidad no parece ser tanto el de definir la naturaleza epistémica del campo inaugurado por Freud, sino el de determinar cuáles son o deberían ser las formas legítimas de investigar en la disciplina.

Ulrich Moser distinguió tempranamente, basándose en términos computacionales, las investigaciones psicoanalíticas "on-line" de las "off-line":

El terapeuta en la práctica psicoanalítica es (...) un investigador on-line (...); esto por dos razones. Primero, el terapeuta, como parte de un sistema, elije al objeto que observa y lo va cambiando permanentemente y, segundo, debe operar "científicamente" en la situación del manejo terapéutico. Él crea conocimientos. (...) pero la validez de su propio quehacer en este área queda supeditado a la certeza intuitiva, un fenómeno - hasta donde yo sé- al que la investigación psicoterapéutica no ha prestado aun atención"1 (Moser, 1989/1992, p. 183).

Mientras que la investigación on-line designa el trabajo que todo terapeuta realiza en el transcurso del tratamiento con el paciente, la investigación off line refiere al trabajo que se realiza en ausencia del paciente y con la aplicación de diferentes métodos a los materiales producidos en la sesión. Esa distinción se ha mantenido y hoy suele hablarse de investigación clínica e investigación extraclínica. En Argentina, David Liberman, de manera independiente y algunos años antes, había arribado a la misma diferenciación:

Existen dos formas de indagar en psicoanálisis: una es la indagación que se realiza con el paciente durante la tarea analítica (esta labor de investigación sabemos que sólo abarca un trecho relativamente corto) y la otra es la indagación del diálogo psicoanalítico en sesiones ya efectuadas (Liberman, 1970, p. 78).

Roger Perrón, más recientemente, sostiene que desde mediados del siglo pasado podemos distinguir dos tipos de acciones referidas a la investigación en el campo del psicoanálisis: "aquellas donde prevalece una actitud clínica, y aquellas que hacen uso de procedimientos formales sistematizados" (Perron, 1999, p. 3). También Rubén Zukerfeld ha planteado, en sintonía con lo anterior, que podemos diferenciar la "investigación psicoanalítica" de la "investigación en psicoanálisis": mientras que la primera remite a la utilización del método psicoanalítico freudiano exclusivamente en la situación analítica, la segunda supone un conjunto diverso de problemas relativos a la realidad clínica, la situación analítica y la producción analítica (Zukerfeld, 2009).

Robert Wallerstein ha sido uno de los analistas que más empeño ha puesto por caracterizar el campo de la investigación off-line, delimitando algunas áreas posibles que trascienden de algún modo u otro el método clínico tradicional:

1. La investigación clínica sistemática de procesos y resultados con enfoque naturalista, mediante instrumentos y métodos de investigación especializados (como grabaciones, escalas y cuestionarios).

La investigación del desarrollo del infante, en cooperación con investigadores de psicología del desarrollo.

La investigación acerca del área de contacto entre el psicoanálisis y la biología, la medicina y la ciencia natural.

La investigación comparada en el área de intersección entre el psicoanálisis y las ciencias sociales, como la antropología, la sociología, la psicología clínica y social, etc.

La investigación de las aplicaciones del psicoanálisis a las humanidades, como la literatura, la crítica literaria, la biografía, la historia, el arte y la música (Wallerstein, 1993). 
A mi modo de ver, la clasificación más simple y consistente es aquella que diferencia tres grandes clases: investigación clinica, investigación conceptual e investigación empirica (Leuzinger-Bohleber y Bürgin, 2003; Leuzinger-Bohleber, 2015):

1. La investigación clínica se origina a partir de los problemas que plantea un sujeto y su trabajo analítico. El analista funciona, en un segundo momento, como investigador: no es durante el trabajo clínico que investiga, sino a posteriori; elabora el caso y teoriza a partir de él. Se trata del proceso de generación de insights y conocimientos, que permite contrastar los modelos teóricos con el material clínico.

La investigación conceptual se refiere al abordaje sistemático y metódico de los significados implícitos y explícitos de los conceptos psicoanalíticos, en sus usos clínicos y extraclínicos. Supone el tratamiento de problemas relativos a la producción, evolución o implantación geográfica de conceptos, así como a la forma en la que los conceptos se vinculan con los referentes, con el nuevo conocimiento o con conceptos de otras disciplinas, etc. (cf. Dreher, 2000, 2005).

Finalmente, la investigación empirica engloba a la indagación extraclínica de las teorías y modelos que han nacido en el contexto clínico, la investigación sobre la eficacia de los tratamientos, etc.

Esta clasificación pareciera haberse impuesto con algún grado de consenso en la comunidad psicoanalítica internacional y es sobre ella que se diseñan actualmente investigaciones en psicoanálisis. La reciente versión de la Revisión a puertas abiertas de estudios de resultados y procesos en psicoanálisis (Leuzinger-Bohleber y Kächele, 2015) profundiza esas mismas categorías a partir de los desarrollos efectuados en diversas partes del mundo, para dar testimonio de ese consenso. El uso de esta taxonomía puede constatarse en el lugar que han venido alcanzando en algunas de las más importantes revistas psicoanalíticas, como el Journal of the American Psychoanalytic Association y el International Journal of Psychoanalysis. En nuestro margen latinoamericano también podemos advertir que el tema de la investigación se ha venido discutiendo en diversos encuentros y presentaciones psicoanalíticas de las últimas décadas, y se han utilizado algunas de esas nominaciones.

Más allá del consenso relativo de esa tripartición, las taxonomías reseñadas son apenas una muestra de las diversas maneras en las que se ha intentado organizar descriptivamente el campo de la investigación en psicoanálisis. Aun con la clasificación alcanzada, persisten dificultades inherentes a la exhaustividad y disjuntividad de las clases utilizadas.

Se ha dicho que las fronteras son las cicatrices de la historia. Pues bien, eso vale también para los confines de la investigación en psicoanálisis. Nos adentraremos ahora en uno de los aspectos posiblemente más controversiales de la investigación en este campo: el debate entre las investigaciones que priorizan el contexto psicoanalítico tradicional y las investigaciones que priorizan otros diseños y metodologías. Primero reseñaremos el surgimiento y caracterización de lo que ha dado en llamarse investigación empírica sistemática, analizando los principales argumentos que intentan promoverla y algunos de los principales ámbitos en los que ha venido desarrollándose. Seguido de ello, examinaremos las principales resistencias que este tipo de investigación ha suscitado en las comunidades analíticas, y haremos especial hincapié en el valor que unos y otros otorgan a la metodología del EC y a su papel en psicoanálisis.

\section{RAZONES PARA LA INVESTIGACIÓN EMPÍRICA SISTEMÁTICA}

En las últimas décadas algunos sectores de la comunidad psicoanalítica se han visto en la necesidad de contrastar y complementar los conocimientos alcanzados por el método clínico tradicional con saberes que pueden surgir con otras metodologías de investigación, a los fines de contribuir a la credibilidad de la disciplina. Así, en 1998 la Asociación Internacional de Psicoanálisis creó un comité de investigación empírica (presidido inicialmente por Robert Wallerstein y luego por Peter Fonagy), que ha venido organizando encuentros, ofertando cursos y financiando proyectos de investigación (Lemlij, 2013). Con la expresión de 
investigación empirica sistemática se suele referir a aquellas investigaciones que no se basan en la metodología tradicional de historiales clínicos ni en diseños experimentales o cuasi-experimentales, sino que constituyen una forma intermedia: aspiran a ser sistemáticas pero no a ser experimentales. Es decir, se orientan por una secuencia invariante de pasos en la que intervienen instrumentos específicos de recolección y/o análisis de datos, junto con criterios formalizados de evaluación, pero sin pretender la manipulación controlada de variables propia de los experimentos.

$\mathrm{Al}$ menos tres aspectos metateóricos merecen ser tenidos en cuenta para comprender la evolución y el espíritu de este tipo de investigaciones. Primero, la asunción, por parte de varios sectores psicoanalíticos, de las críticas epistemológicas a la validez de los conocimientos obtenidos mediante la investigación clínica; fundamentalmente aquellas objeciones encabezadas por el inductivismo eliminativo de Adolf Grünbaum (1984; 1993). Segundo, la afinidad metateórica que este sector del psicoanálisis ha venido mostrando con las ciencias hegemónicas; naturalismo que los ha orientado hacia la búsqueda de otros caminos para dar cuenta de nuestro campo de fenómenos: se han propuesto diversos procedimientos, provenientes de diferentes disciplinas consolidadas, para enmendar las denunciadas carencias de objetivación y sistematización del procedimiento tradicional. El tercer aspecto no es menos importante: el desarrollo de esta área de investigación se comprende mejor si tenemos en cuenta que las críticas epistemológicas al psicoanálisis empalmaron, durante la década de los 80, con la retirada del Estado Benefactor, cuya seguridad social incluía, en algunos países europeos, la cobertura de tratamientos psicoanalíticos. Por esta época se iniciaron las demandas al psicoanálisis sobre su "eficacia terapéutica", a los fines de justificar el coste de los tratamientos, que ahora tenían que competir con otras formas consolidadas de abordajes psicoterapéuticos. Al mismo tiempo, la llegada de lo que ha dado en llamarse medicina basada en evidencias al campo de la salud mental, ha favorecido una toma de conciencia en ciertos sectores psicoanalíticos de que la investigación empírica sistemática es necesaria y de que prescindir de ella constituye una amenaza al porvenir del psicoanálisis como ciencia y como terapéutica (Bornstein, 2001; Fonagy, 2000; Gunderson y Gabbard, 1998; Kächele, 1992; Safran, 2001). Este tipo de exigencia, proveniente del exterior al movimiento psicoanalítico, contribuyó a las reorganizaciones de la IPA, por ejemplo. La publicación, en 1999, del Open Door Research Review, contenía las principales ideas directrices sobre qué respuesta dar a tales demandas. Cabe destacar que la postura crítica de varios de sus miembros (como André Green y Roger Perron) derivó en la creación del segundo subcomité de investigación conceptual, clínica, epistemológica e histórica, lo que dio lugar a voces disidentes respecto de la investigación empírica que proponían los naturalistas para dar respuesta a las demandas externas.

La muestra más contundente de la incidencia fundamental que las críticas epistemológicas y metodológicas han tenido en el desarrollo de estos debates la constituye el hecho de que han sido internalizadas: mientras que inicialmente la contienda de los psicoanalistas era mantenida con adversarios externos (generalmente filósofos de la ciencia o psicólogos de otras orientaciones, pero que presentaban objeciones epistemológicas), actualmente el campo de batalla es la comunidad psicoanalítica misma. Allí se dirimen las discusiones inherentes a la validación teórica, quedan representadas las posturas ya existentes y se asumen muchas demandas de legitimidad como requisitos autoimpuestos.

La conclusión metodológica más importante que un sector de la comunidad psicoanalítica (de orientación naturalista ${ }^{2}$ ) ha venido extrayendo de las principales críticas epistemológicas es, posiblemente, la objeción al EC como metodología prínceps para la investigación psicoanalítica. Adolf Grünbaum ha sido uno de los críticos que más insistencia ha puesto en esta idea: "si el psicoanálisis tiene futuro como una empresa científica, es muy probable que no radique en el método de historia clínica, sino con otros diseños de prueba”3 (Grünbaum, 1988, p. 657). No son pocos los analistas que se hicieron eco de esta crítica. Peter Fonagy, quien se ha ocupado agudamente del tema, afirma que

Hay tres condiciones que deberían cumplirse para que la investigación clínica sea una metodología adecuada y pueda ser la única metodología aceptada en la construcción de teorías psicoanalíticas. Estas son: a) una conexión lógica estrecha entre 
la teoría y la práctica, b) un razonamiento deductivo apropiado en relación al material clínico, y c) el uso no ambiguo de términos ${ }^{4}$. (Fonagy, 1999, p. 13)

Fonagy considera que ninguna de esas tres condiciones se cumple y concluye que

El estudio de casos, en sí mismo, sin embargo, es insuficiente como método de investigación. Precisa ser complementado por otros procedimientos confirmatorios tales como la replicación, los estudios experimentales detallados y las investigaciones anatómicas, genéticas y neurofisiológicas 5 (Fonagy, 1999, p. 20).

Helmut Thomä y Horst Kächele, en coincidencia con ello, piensan que ha habido una frecuente desestimación u omisión de los aspectos evaluativos de la teorización emergente en los estudios de caso:

Los analistas más influyentes mantienen una actitud de condescendencia hacia la investigación sistemática. Se sienten satisfechos con generar hipótesis dentro de la situación clínica. La diferencia entre el contexto de descubrimiento y el contexto de justificación parece ser desconocida para esos analistas que se contentan con la descripción de resúmenes de casos clínicos (Thomä y Kächele, 1999/2000, p. 102).

En ese sentido, para estos autores "debemos restringir la investigación psicoanalítica aludida en la tesis [freudiana] de la unión inseparable, en el sentido de que sus resultados pueden ser usados sólo para el descubrimiento y el desarrollo de hipótesis preliminares, y no para su verificación” (Thomä y Kächele, 1985/1989, p. 519; corchetes añadidos). Además, consideran que

...es una exigencia muy ambiciosa querer unir la investigación psicoanalítica en la situación analítica (entendiendo por tal
la obtención de nuevas hipótesis psicoanalíticas, que se debe distinguir estrictamente de la investigación para verificar tales
hipótesis a través de terceros no comprometidos en el tratamiento) con los intereses curativos. Esta exigencia no podrá ser
satisfecha, si el analista no distingue, en la teoría de la técnica, los siguientes componentes independientes: curación, obtención
de nuevas hipótesis, prueba de las hipótesis, exactitud de las explicaciones y utilidad del conocimiento.

En términos generales, los partidarios de la investigación empírica han venido sosteniendo la necesidad de utilizar los resultados de las investigaciones clínicas y conceptuales para desarrollar investigaciones extraclínicas, pues consideran que sólo en estas últimas radica la posibilidad de justificar los desarrollos teóricos del psicoanálisis.

Robert Wallerstein $(2001,2006)$ efectúa un análisis de la evolución de las investigaciones empíricas sobre procesos y resultados, caracterizado por la existencia de diversas generaciones,que representan el espíritu predominante de este tipo de producciones en distintos momentos históricos. Se puede atisbar en esa descripción cómo el comienzo de estas investigaciones en la década de 1920, signado por las necesidades de determinar el grado de éxito terapéutico, procedía con criterios bastante inespecíficos y mediante rudimentarios informes estadísticos de estudios retrospectivos. Pero también cómo fueron abriéndose paso, paulatinamente, estudios formales y sistemáticos en los que se combinaron los análisis de procesos con los análisis de resultados psicoterapéuticos, para culminar, desde 1970 en adelante, tanto en la utilización de nuevas tecnologías para la recolección y análisis de los datos ${ }^{6}$, así como en la creación de métodos específicos para la investigación en el campo. Entre las metodologías más conocidas diseñadas para investigar empírica y sistemáticamente aspectos de la situación analítica, podemos mencionar el Método del Tema Central de Conflicto Relacional (CCRT) ideado por Lester Luborsky (1976, 1977, 1990), el método de las Estructuras Emocionales Fundamentales Repetitivas y Desadaptadas (FRAMES) de Dahl (1988, 1998), la teoría de los Códigos Múltiples de Wilma Bucci (1997), el Procedimiento de Evaluación Shedler-Westen (SWAP) de Jonathan Shedler y Drew Westen (1998, 1999), el método PQS de Enrico Jones (2000), el método del Plan de Acción Latente del Terapeuta (TLAP), diseñado por Ávila-Espada y Mitjavila (2003); el Algoritmo David Liberman (ADL) desarrollado por Maldavsky (2004) y el Modelo de los Tres Niveles para la Observación de las Transformaciones del Paciente (3-LM), ideado por Ricardo Bernardi (2015). La variabilidad de problemas y procedimientos a los que estos métodos se aplican hace imposible establecer algún denominador común característico, más allá del mencionado hecho de que se sostiene la necesidad de ir más allá de la 
metodología basada exclusivamente en la indagación clínica on-line. Esto no supone necesariamente una completa desestimación del estudio de casos, pero sí la suposición de que es necesario trascenderlo, por ejemplo combinando la investigación clínica con un tratamiento sistemático de la información obtenida.

\section{EXPERIMENTACIÓN Y NEUROPSICOANÁLISIS}

Coincidimos con Leuzinger-Bohleber (2015) en que conviene diferenciar subtipos de investigación extraclínica a partir del diseño utilizado, ya que, además de las mencionadas empíricas y sistemáticas, han ganado terreno las investigaciones experimentales e interdisciplinarias, con marcadas características propias.

En las últimas décadas ha habido un creciente número de investigaciones que demuestran la contrastabilidad de las hipótesis psicoanalíticas, y que arrojan significativa evidencia empírica a su favor (Bornstein y Masling, 1998a, 1998b; Fisher y Greenberg, 1996; Kline, 1972; Sarnoff, 1971; Shapiro \& Emde, 1995; Westen, 1999). En lo que va del siglo XXI, el camino más promisorio en ese sentido pareciera ser, para algunos analistas, el de la relación del psicoanálisis con las neurociencias.

Eric Kandel, premio Nobel de Fisiología o Medicina en el 2000, ha sido uno de los promotores de la investigación psicoanalítica a partir de su relación con otras disciplinas, particularmente las neurociencias. Kandel ha afirmado que

El futuro del psicoanálisis, si es que tiene un futuro, está en el contexto de una psicología empírica, apoyada en técnicas de imagen, métodos de neuroanatomía, y genética humana. Engastado en las ciencias del conocimiento humano, las ideas del psicoanálisis pueden ser testeadas, y es así que esas ideas pueden tener su mayor impacto ${ }^{7}$ (Kandel, 1998, p. 168).

Kandel considera que el psicoanálisis "sigue representando la visión más coherente e intelectualmente satisfactoria de la mente"8 (Kandel, 1999, p. 505), pero mantiene una opinión desfavorable sobre el método clínico: "como herramienta de investigación, este método concreto ha agotado su poder. Cien años después de su creación (...) es necesario reconocer que (...) no proporciona una base suficiente a la ciencia de la mente" (Kandel, 1999, p. 506). ¿No es acaso curioso que "la visión más coherente e intelectualmente satisfactoria de la mente"se haya basado en un procedimiento mágicamente agotado?

En el año 2000 fue fundada la Sociedad Internacional de Neuropsicoanálisis y creada la primera revista destinada a ello, llamada Neuro-Psychoanalysis. Mark Solms y Oliver Turnbull, pioneros en este intento de relación, consideran que los afanes investigativos de Freud se vieron limitados por el desarrollo tecnológico de su época, pero que

...nosotros ahora tenemos métodos neurocientíficos que nos permiten estudiar la naturaleza dinámica de la mente para identificar la organización neuronal de su subestructura inconsciente (...) Freud, en nuestra opinión, habría considerado esto como un desarrollo completamente legítimo y bienvenido de su trabajo pionero ${ }^{10}$ (Solms \& Turnbull, 2011, p. 135).

Estos autores manifiestan su acuerdo con la perspectiva freudiana del conocimiento como modelo de la realidad y afirman que "la neurociencia ofrece una segunda perspectiva de la 'cosa' incognoscible que denominamos aparato mental, la cosa que Freud intentó describir por primera vez en su metapsicología"11 (p.137). Algunos de los principales méritos de este enfoque han sido la integración de tratamiento psicoanalítico a la investigación de pacientes neurológicos (Kaplan-Solms \& Solms, 2000/2005) ${ }^{12}$ y la corroboración empírica de la teoría freudiana de los sueños más allá del ámbito clínico (Solms, 2001).

A pesar de que son conocidas las dificultades que conlleva utilizar diseños experimentales para testear las hipótesis psicoanalíticas, como, por ejemplo, los problemas de operacionalizar conceptos de carácter metafórico y sumamente polisémicos (Fonagy, 1982), en las últimas décadas eso ha sucedido de una manera considerable. Las investigaciones de Howard Shevrin sobre el procesamiento preconsciente e inconsciente de la información en la memoria y los sueños, constituyen un buen ejemplo de ello. Shevrin ha investigado durante más de 40 años en la intersección del psicoanálisis con las neurociencias y la psicología cognitiva, 
fundamentalmente buscando evidencia experimental de los conceptos freudianos. Luego de asumir que diversos fenómenos psíquicos pueden ser observados y redefinidos como magnitudes físicas de la actividad cerebral, ha indagado en el terreno compartido entre psicoanálisis y neurobiología. En 1968 dio a conocer la primera investigación sobre respuestas cerebrales a estímulos visuales inconscientes, y arguyó que ello proveía una fuerte evidencia objetiva sobre la existencia de procesos inconscientes (Shevrin \& Fritzler, 1968). En los últimos años, Shevrin aportó nuevos datos que corroboran un vínculo causal entre la noción de conflicto inconsciente y los síntomas experimentados por sujetos con trastornos de ansiedad; por ejemplo, fobias. Él mismo ha reconocido que su investigación ha sido motivada por la crítica de Grünbaum: "en respuesta a estos desafíos, hemos aplicado nuestro método de investigación con el fin de encontrar pruebas independientes para la validez de las construcciones de la represión y el conflicto inconsciente"13 (Shevrin et al., 2013, p. 2).

El espíritu de estas investigaciones interdisciplinarias ha sido sintetizado coherentemente por Juan Pablo Jiménez:

La confianza absoluta -y excluyente- en el método clínico como forma de validación ha tenido importantes consecuencias para el desarrollo de la teoría y de la práctica psicoanalítica, las que se pueden resumir en una sola palabra: aislamiento. El aislamiento autoimpuesto, tanto de las ciencias médicas como de las psicológicas, es quizás el mayor obstáculo para que el psicoanálisis se haga un lugar en la mesa de la academia del siglo veintiuno (Jiménez, 2004).

También Fonagy denuncia el “espléndido aislamiento” del psicoanálisis respecto de las ciencias de nuestra época:

Mientras que el psicoanálisis clínico necesita poca ayuda para llegar a conocer la subjetividad de un individuo del modo más detallado posible, cuando desea generalizar a un modelo global de la mente humana, la disciplina no puede existir por sí sola. El hecho de que hace 50 años no hubiera otros campos de investigación relativos a la mente que ofrecieran una información merecedora de integrarse con el modelo psicoanalítico, es probablemente la raíz de nuestro encomiable esfuerzo por "recorrer el camino solos". Pero la escena científica ha cambiado radicalmente en las dos últimas décadas. Para decirlo en otras palabras, si bien se llega a conocer mejor la mente de un individuo en un marco de trabajo exclusivamente psicoanalítico, el modelo psicoanalítico general de la mente, si es que existe, debería alinearse con el conocimiento más amplio de la mente conseguido gracias a un abanico de disciplinas. Y esto ya está sucediendo, si bien es cierto que de manera informal (Fonagy, 2003, p. 218).

Fonagy considera que la investigación interdisciplinaria no puede testear la teoría psicoanalítica, pues "no puede demostrar que unas ideas psicoanalíticas determinadas son verdaderas o falsas” (2003, p. 11), pero considera que sí puede contribuir a "elaborar los mecanismos mentales que se ponen en funcionamiento para generar los fenómenos que describen los escritos psicoanalíticos” (p. 11). Es en ese sentido que

...el psicoanálisis podría beneficiarse si integrara sus teorías con los hallazgos de investigación de otros campos elaborando los modelos psicológicos psicoanalíticos de los mecanismos implicados en los procesos mentales claves. Esto ayudaría a su vez a sistematizar nuestra base de conocimiento de modo que esa integración con las nuevas ciencias de la mente se volviera cada vez más fácil. No sólo seríamos capaces de mostrar más fácilmente que nuestro tratamiento funciona, sino que tendríamos nuevas posibilidades de comunicar nuestros hallazgos a otros científicos (p. 12).

Fonagy examina el caso de las investigaciones en genética del comportamiento y cómo el psicoanálisis podría aportar a las discusiones actuales, pues puede elucidar el “eslabón perdido en la cadena de la explicación que va desde los genes a la personalidad y la conducta” (p.18), a saber, la forma en la que los sujetos interpretan su experiencia en el mundo: la realidad psíquica. Sugiere que las formas de constituir la realidad psíquica determinan la expresividad o no de ciertos genes, como parecen insinuar los estudios sobre el apego.

Más allá de los intercambios específicos, Fonagy cree que el "aislamiento autoimpuesto de los psicoanalistas" es autodestructivo:

...privarnos de oportunidades de colaborar en el progreso científico podría dar lugar muy pronto a una pérdida injustificada de credibilidad científica e incluso, algo relacionado con esto pero más trágico todavía, a la pérdida de nuestra respetabilidad profesional en el mercado clínico dominado por la ciencia (p. 18). 
Una pregunta que ha cobrado forma en el seno de estos debates es si puede el psicoanálisis entablar un diálogo productivo con las neurociencias sin poner en riesgo su propia perspectiva. Aunque muchos analistas parecen apreciar los intercambios interdisciplinarios con las neurociencias, hay quienes expresan sus inquietudes respecto a un posible efecto deletéreo sobre la teoría y la práctica psicoanalíticas. Varios de los promotores de este tipo de desarrollos, dentro de la tradición naturalista, piensan que tales intercambios pueden ser necesarios (aunque no sean suficientes) para la viabilidad futura del psicoanálisis (Yovell et al., 2015). Vemos, una vez más, la presencia de un cierto afán de viabilidad como motor de estos programas de investigación en psicoanálisis; cuyo supuesto de partida es el de una inquietante inviabilidad antecedente, originada fundamentalmente por las críticas epistemológicas y sobre un mismo núcleo de aspectos problemáticos. Todo esto a pesar de que formalmente se reconozca que "la neurociencia ya no es el tribunal de apelación final para el psicoanálisis, así como tampoco el psicoanálisis lo es para la neurociencia. El último tribunal de apelación para los psicoanalistas es la situación clínica" ${ }^{14}$ (Solms, 2013, p. 18; cursiva del original).

\section{LAS INVESTIGACIONES SOBRE LA EFECTIVIDAD DEL PSICOANÁLISIS}

El asunto de la efectividad comparada de las distintas psicoterapias ha sido y sigue siendo uno de los tópicos más controversiales en diversos campos de la psicología clínica. En psicoanálisis han sido los partidarios de la tradición naturalista los que se han ocupado sistemáticamente del tema, dando cuenta de lo que podríamos llamar la contracara clinica de las mismas pretensiones de viabilidad epistemológica.

Entre las diversas razones por las que se han venido promoviendo y realizando estudios sobre la eficacia del psicoanálisis en las últimas décadas en el seno de la IPA se destaca la necesidad de persuadir a los sistemas de salud de la necesidad de financiar los tratamientos psicoanalíticos (Lancelle, 1997), sobre todo cuando hay competidores cada vez mejor establecidos: "cuando diversos métodos terapéuticos se ofrecen como alternativas más rápidas, menos costosas y más eficaces que el psicoanálisis, el estudio de la eficacia del análisis se convierte en un desafío para los psicoanalistas" (Rubistein y Trotta, 2004). Estos problemas, que en principio parecieran poder separarse de los asuntos de los que tradicionalmente se ha ocupado la epistemología, son vinculados una y otra vez con los problemas relativos a la legitimidad de la disciplina:

La llamada "paradoja de la equivalencia" - según la cual hasta el momento no ha sido posible demostrar la superioridad terapéutica de algún tipo de psicoterapia-, refuerza la afirmación de Grünbaum de que el psicoanálisis no ha aportado ninguna prueba de que sus resultados terapéuticos se deban a la verdad de las interpretaciones dadas a los pacientes y no al efecto placebo (Jimenez, 2004).

Actualmente disponemos de evidencia contundente sobre la efectividad del psicoanálisis y de las distintas variedades de psicoterapias psicoanalíticas. Jonathan Shedler, en un interesante artículo de revisión, especifica que contamos con datos "que demuestran que los beneficios terapéuticos de sujetos que han recibido terapia psicodinámica no sólo se mantienen, sino que además hacen que los pacientes sigan mejorando al cabo del tiempo" 15 (Shedler, 2010, p. 98). En el mismo trabajo, este autor plantea que hay evidencia de que "las terapias no psicodinámicas pueden ser efectivas en parte porque los terapeutas más experimentados utilizan técnicas que durante mucho tiempo han sido elementos centrales de la teoría y la práctica psicodinámica"16 (p. 18).

Las investigaciones de los últimos años comprueban que la eficacia de las psicoterapias psicoanalíticas tiene equivalente magnitud de efecto terapéutico que otras psicoterapias que suelen catalogarse como basadas en la evidencia o apoyadas empiricamente. Shedler comenta su impresión al recabar la información sobre los metanálisis de estudios de eficacia:

En la redacción de este artículo me han sorprendido diversas ironías. Una es que los académicos que rechazan los enfoques psicodinámicos, a veces vehementemente, con frecuencia lo hacen en nombre de la ciencia. Algunos defienden una ciencia 
de la psicología fundamentada exclusivamente en el método experimental. Y pese a ello, el mismo método experimental llega a conclusiones que apoyan los conceptos y tratamientos psicodinámicos ${ }^{17}$ (Shedler, 2010, p. 106).

Shedler se asombra también del hecho de que la mayoría de los psicoanalistas clínicos no estén familiarizados con los resultados generales de tales estudios sobre la eficacia: "del mismo modo que el sentimiento antipsicoanalítico puede haber impedido la divulgación de esta investigación en los círculos académicos, igualmente la desconfianza en los métodos de investigación académica puede haber impedido su divulgación entre los círculos psicoanalíticos"18 (p. 106). Sin embargo, Shedler sostiene que la mayoría de los informes de resultados de investigación sobre estudios de eficacia terapéutica están escritos en un lenguaje poco accesible para los analistas clínicos: son densos, complejos y muy técnicos, y con frecuencia parecen haber sido escritos principalmente para otros investigadores de psicoterapia y no para los clínicos. Todo esto redunda en un escaso o nulo aprovechamiento de los resultados.

Peter Fonagy comienza un artículo reciente destinado al tema afirmando que "la terapia psicodinámica (PDT) está en retirada en todo el mundo, ante las críticas de su credibilidad científica” (Fonagy, 2015, p. 137). Esto nos deja entrever nuevamente que el interés por este tipo de estudios se origina en las demandas epistemológicas mencionadas. Fonagy considera allí que la mayoría de los metanálisis utilizados (que carecen de datos de pacientes individuales y se basan en tasas de respuesta y valores medios) para ponderar la significación general de la eficacia terapéutica encierran un problema crucial: pueden ser engañosos cuando los estudios aleatorizados controlados que se toman en cuenta no son homogéneos en cuanto a la población elegida como objetivo, el método de tratamiento y los criterios de valoración (p. 137). Este suele ser el caso de los estudios sobre la eficacia de las terapias psicoanalíticas ${ }^{19}$. Otra de las dificultades señaladas por Fonagy es en torno a la definición misma de psicoterapia psicodinámica, pues el conjunto que este término designa se ha vuelto cada vez más heterogéneo y sus límites cada vez más difusos.

Luego de analizar una serie de metanálisis, Fonagy concluye su artículo afirmando que para hacer avanzar este campo, no alcanza con reflexionar sobre la eficacia general de una categoría heterogénea de enfoque psicoterapéutico aplicado a un grupo heterogéneo de pacientes (por ejemplo, los que experimentan depresión), sino que se necesita una sistemática modularización de las intervenciones posibles y su interrelación, en combinación con las necesidades de pacientes individuales, a los fines de poder indagar coherentemente la eficacia ${ }^{20}$.

Algunos analistas francófonos también han sabido advertir el valor que implica conocer sobre la eficacia del psicoanálisis. Colette Soler, por ejemplo, no duda en afirmar que la prevalencia dada al dispositivo analítico se justifica porque

...hay un éxito del psicoanálisis, es decir que hay logros terapéuticos: eso nos asegura, y eso le aseguró de entrada a Lacan, que hay un real en juego en este dispositivo. Por eso no hay que hablar con demasiado desprecio de los logros terapéuticos, porque, por empezar, no hay psicoanálisis sin éxito terapéutico, que aunque parcial es ciertamente necesario, y porque si no hubiera efecto terapéutico, eficiencia terapéutica, no quedaría, después de todo, nada del psicoanálisis, no quedaría nada para aseguramos de que todo ese bla-bla está conectado con algo real (Soler, 1988/2007, p. 15).

Aunque dudo mucho de que Soler y Fonagy se pudiesen poner de acuerdo sobre qué entender por eficacia y qué por éxito terapéutico, lo cierto es que gran parte de lo que motoriza el desafío de los estudios de eficacia de nuestra práctica, asumido por muchas comunidades analíticas, pareciera derivar, implícita o explícitamente, de los problemas epistemológicos y metodológicos que han promovido e instalado los grandes movimientos críticos en la disciplina.

\section{LAS RESISTENCIAS A LA INVESTIGACIÓN EMPÍRICA}

La relación entre la investigación empírica sistemática extraclínica y el trabajo que realiza la mayoría de los psicoanalistas ha sido siempre controvertida. Resulta muy sugerente el título de un trabajo de Peter 
Fonagy, "Agarrando la ortiga: o de por qué la investigación psicoanalítica es tan irritante"21 (2000), pues alude metafóricamente a la difícil situación en la que se hallan los psicoanalistas dedicados a la investigación, habitualmente frustrados y desestimados por sus colegas clínicos, quienes rechazan vehementemente la naturaleza de su trabajo y sus resultados. Hay, al decir de Otto Kernberg (2006), una notoria "ansiedad de investigación" en la comunidad psicoanalítica, debido a las inquietudes que ella provoca.

Jonathan Shedler (2002) ha examinado el escepticismo con que los analistas ven a la investigación empírica y ha distinguido tres tipos de objeciones principales: que es imposible, que es innecesaria y que es irrelevante. Shedler cree que la primera objeción puede ser la opinión prevalente, pues debido a los intentos frustrados de vincular la investigación empírica con el ámbito de trabajo clínico, muchos se han sentido tan decepcionados por los resultados que han abandonado la esperanza:

No ven modo de cuantificar las construcciones psicoanalíticas importantes sin trivializarlas y distorsionarlas. Les desanima el tipo de reduccionismo que ha caracterizado la investigación empírica en el pasado. A partir de la observación "no se ha hecho" han razonado "no puede hacerse"22 (Shedler, 2002, p. 430).

La segunda objeción (que la investigación empírica es innecesaria) proviene de aquellos analistas que consideran al método analítico como algo necesario y suficiente para generar, validar y revisar la teoría. Shedler afirma que uno de los problemas de esta consideración es que décadas de investigación analítica tradicional no han podido resolver las diferencias teóricas que dividen a nuestra disciplina:

Tenemos modelos que compiten entre sí con diferentes implicaciones para la técnica y los defensores de cada uno de esos modelos publican regularmente estudios de casos que "demuestran" la superioridad de su enfoque. Los estudios de casos no parecen hacer cambiar a nadie de opinión, porque los analistas de diferentes tendencias ven cosas diferentes en un material clínico prácticamente igual $^{23}$ (Shedler, 2002, p. 430).

Las creencias teóricas de los analistas parecen apoyadas más en la identificación con quienes estuvieron a su cargo en su formación, con sus supervisores y con su propio analista, que en la significación de la evidencia empírica. A su vez, sostiene Shedler, las pruebas obtenidas son mayoritariamente a partir de casos clínicos, cosa que ha servido como evidencia sólo para los propios analistas y que ha sido insuficiente para los interesados provenientes de otras disciplinas (p. 431).

La tercera objeción (que la investigación empírica es irrelevante) es mantenida por aquellos analistas que piensan que las reglas de la ciencia no se pueden o no se deben aplicar al psicoanálisis, dado que su intención central ha sido sostener que el psicoanálisis es hermenéutica, no ciencia (p. 431). Una versión postmoderna de esta postura proviene de las corrientes intersubjetivistas -según Shedler-, que consideran al encuentro psicoanalítico como una construcción intersubjetiva en la que el analista y el analizando construyen juntos una realidad única. Pero,

Por mucho que valoremos la singularidad de nuestros pacientes, no nos aproximamos a ellos en un vacío. Si tenemos experticia en el tratamiento del sufrimiento humano, es precisamente porque podemos reconocer aspectos comunes en los pacientes y comprender su sufrimiento en un contexto más amplio de patrones y síndromes conocidos ${ }^{24}$ (Shedler, 200, pp. 2431-432).

Como podemos notar, en las objeciones que Shedler ubica en el escepticismo hacia la investigación empírica aparecen involucrados varios aspectos de diferente índole: desde objeciones pesimistas basadas en la experiencia previa hasta argumentos basados en asunciones epistémicas (no siempre explicitadas) respecto a la naturaleza del conocimiento psicoanalítico.

Los defensores del método clínico tradicional para la investigación en psicoanálisis tienen, sin embargo, una mirada diferente de estas cuestiones. 


\section{LA DEFENSA DEL MÉTOdo CLÍNiCo PSiCOANALÍTiCo PARA INVESTIGAR: REVISión de ALGUNOS PRESUPUESTOS}

Los partidarios de la investigación clínica parten de una crítica a la concepción de ciencia preponderante en los enfoques naturalistas de investigación, a la vez que sostienen que el procedimiento tradicional de estudio de casos es el único método apropiado para investigar y desarrollar el psicoanálisis (Green, 1996; Hoffman, 2009; Lothane, 1998; Nasio, 2001; Perron, 1999; Wolff, 1996).

Según Roger Perrón, la investigación clínica tiene tres objetivos fundamentales. En primer lugar, en sintonía con la investigación clínica de la medicina y la psiquiatría, apunta a definir síndromes psicopatológicos, a establecer y a revisar categorías nosológicas. En segundo lugar, se propone formular construcciones teóricas: es la investigación clínica la que ha permitido el desarrollo de los grandes modelos teóricos en psicoanálisis, incluso después de Freud. En tercer lugar, la investigación clínica aspira a proveer fundamentos conceptuales para el abordaje terapéutico (Perron, 1999, p. 4).

Los defensores de esta metodología sostienen que éste fue el camino que Freud siguió para investigar, por el que llegó a establecer modelos de funcionamiento de la neurosis obsesiva, la histeria, la fobia y la paranoia, genialmente ejemplificados con sus conocidos casos ejemplares. Muchos afirman que éste debe seguir siendo el recurso esencial de toda investigación psicoanalítica y que no hay necesidad alguna de buscar vías alternativas. Por otro lado, intentar cumplir con varios de los requisitos metodológicos ideados para evaluar la investigación en ciencias naturales no contribuye sino a una destrucción del objeto de estudio psicoanalítico. Además de ello, tales requisitos (cuantificación de las variables, replicabilidad de la observación, posibilidad de predicción, etc.), lejos de ser universalmente aceptados, han sido discutidos desde diversas perspectivas filosóficas de las ciencias naturales, lo cual resta razonabilidad a su extrapolación indiscutida al psicoanálisis.

Se ha afirmado que toda investigación fuera de la situación psicoanalítica, por ejemplo, la investigación cuasiexperimental de las teorías, resulta imposible porque no puede captar la complejidad de los conceptos y modelos psicoanalíticos en situación natural y, por ello, resulta irrelevante para el psicoanálisis y su práctica (Masling y Bornstein, 1996; Westen, 2002; Shedler, 2002). Por lo tanto, para los partidarios del enfoque clínico, la tesis freudiana de la unión inseparable entre curar e investigar, mantiene su vigencia.

André Green $(1996,2000)$ no ha vacilado en sugerir que la investigación extraclínica, en tanto que atenta contra la pureza del psicoanálisis, puede resultar peligrosa: sólo el método tradicional de EC puede captar las complejidades de los modelos psicoanalíticos sin distorsionarlas o trivializarlas.

Por otro lado, algunos hacen hincapié en el instrumentalismo que deben tener los modelos metapsicológicos, lo cual vuelve absurda su pretendida ampliación experimental. Algunos como Roy Schafer (1976) y Donald Spence (1984), por ejemplo, han defendido la perspectiva de atenerse a una verdad narrativa para entender el valor de los hechos clínicos, desestimando por ello toda búsqueda de relaciones nomológicas relativas a la conducta.

Aunque no voy a analizarlo en detalle, vale la pena mencionar que la comunidad psicoanalítica ha asistido a una serie de poémicas sumamente ilustrativas a propósito de este debate: el debate de Green (2005/2006) con Wallerstein (2005/2006) y Stern (2000) por un lado y, más recientemente, la polémica de Hoffman (2009) con Eagle y Wolitzky (2011) y Safran (2010). La lectura de tales controversias permite entender la posición de unos y otros respecto de cómo debería desarrollarse la investigación en la disciplina, pero, fundamentalmente, permite comprender algunos de los presupuestos inherentes al conocimiento psicoanalítico que los distintos actores mantienen en la elaboración de sus argumentos. Más allá de estas controversias en particular, considero necesario revisar algunas suposiciones generales que comúnmente son asumidas en los argumentos que se esbozan desde ambos bandos y que resultan difíciles de aceptar.

En primer lugar, quienes rechazan el método de EC como herramienta viable para investigar en psicoanálisis, casi siempre apoyan sus argumentos sobre un modelo idealizado (y no siempre explicitado) 
de las ciencias naturales experimentales, deudor de presupuestos ontológicos, metodológicos y axiológicos cuya pretensión ecuménica no está exenta de dificultades. Además, ese rechazo del EC descansa en una serie de objeciones que exceden el ámbito del psicoanálisis y que parecieran empalman con un debate metodológico más amplio; debate que se ha venido dando fundamentalmente, aunque no exclusivamente, en el campo de la investigación en ciencias sociales (Archenti, 2007; Flyvbjerg, 2004; Stake, 1999; Pérez Serrano, 1994; Simons, 2011; Yin, 2002). Así, las principales de estas objeciones, reencontrables en el interior del psicoanálisis, serían las siguientes: un EC

a) No permite generalizar sus resultados, puesto que el análisis de un solo ejemplo de fenómenos no es representativo de la clase entera y, por ende, no podría proporcionar información confiable sobre la misma.

No tiene relevancia en el contexto de justificación, aunque sí en el de descubrimiento.

No permite un control apropiado de los sesgos del investigador, por lo que arroja un conocimiento excesivamente subjetivo.

Estas afirmaciones -apenas algunas de las que suelen hacerse- siguen siendo analizadas y discutidas en la actualidad metodológica de las ciencias sociales. En los debates psicoanalíticos sobre sus metodologías de investigación, estas afirmaciones son admitidas casi siempre sin discusión por los adherentes a la tradición naturalista, que proclaman la utilización de otras metodologías. Los defensores del método psicoanalítico tradicional no siempre han reparado en el miramiento crítico que algunos desarrollos metodológicos contemporáneos (sobre todo de corte cualitativo) han tenido respecto de estas sentencias. Me parece que, a pesar de las diferencias entre las investigaciones cualitativas y las investigaciones psicoanalíticas basadas en el método clínico, convendría tener en cuenta algunos de sus principales argumentos para quedar mejor posicionados en la disputa que se desarrolla en el interior de nuestro campo. Por eso, voy a recuperar mínimamente algunos de esos argumentos críticos para luego conjeturar sobre su importancia potencial para nuestro campo.

Pasemos ahora a examinar los presupuestos que antes señalé.

\section{I. ¿No puede haber generalización válida a partir de los EC?}

El filósofo danés Ben Flyvbjerg, en un texto ya clásico, analiza cinco malentendidos acerca de la investigación mediante estudios de casos, entre los cuales se encuentran los que he mencionado. Respecto de la supuesta imposibilidad de generalizar a partir del EC, Flyvbjerg nos recuerda que importantes generalizaciones se han perpetrado en la historia de las ciencias a partir del establecimiento de un solo caso. Cita como ejemplo el rechazo a la ley aristotélica de gravedad propuesto por Galileo, quien en su afán por demostrar que todos los objetos caen a la misma velocidad en el vacío con independencia de su peso no se basó en una amplia ni cuantiosa serie de observaciones aleatorizadas, sino fundamentalmente en un experimento conceptual al principio y, más tarde, en uno práctico (Flyvbjerg, 2004). En lugar de continuar por la inagotable vía de los ejemplos, quisiera detenerme en la distinción que realiza Robert Yin (2002, p. 10) entre generalización estadística y generalización analítica: mientras que la primera implica una enumeración de las frecuencias a partir de inferencias muestrales estadísticamente representativas, la segunda supone la expansión a otros casos de teorías o modelos que han permitido entender exitosamente un caso determinado. Lo generalizable es el modelo conceptual que ha permitido obtener singulares resultados en un caso, y no esos resultados específicos ${ }^{25}$. El propio Yin cita y examina casos en donde estas generalizaciones se han efectuado de manera exitosa en ciencias (p. 10). Si nos atenemos a la historia de los descubrimientos científicos, la gran mayoría de ellos se ha efectuado con observaciones intensas posibilitadas por estudios de caso en profundidad y no, como podría creerse, a partir de estadísticas aplicadas a grandes poblaciones.

La generalización a partir del EC suele efectuarse sin problemas en los llamados casos típicos o instrumentales (Stake, 2013), es decir, aquellos que, en función del fenómeno investigado, son similares en aspectos cruciales a otros que hubieran podido seleccionarse. La medida en que los hallazgos de un caso 
puedan generalizarse a otros de la misma clase dependerá, naturalmente, del grado en que los fenómenos bajo estudio sean semejantes en los diversos casos.

Otra forma de generalización posible es a partir de lo que se suelen llamar casos atípicos (que se suelen abordar de forma intrínseca según la terminología de Stake), es decir, aquellos que presentan propiedades excepcionales que se prestan bien a una evaluación crucial de conjeturas, ya que suelen permitir un mejor acceso a ciertas conexiones causales significativas. Piénsese, por ejemplo, en casos como Víctor el niño "salvaje" hallado en Aveyron y su abordaje por parte de Jean Itard. En su momento se pensó, más allá de todo lo que se ha investigado después, que la supuesta ausencia de sociabilidad de este niño permitiría entender el papel que ella juega en el desarrollo moral ${ }^{26}$.

Por otro lado, además de lo anterior, hay que decir que no siempre el tipo de generalización buscada tiene que ser universal: a sabiendas de que muchas de las variables construidas en ciencias de lo humano son contextodependientes, entonces la generalización mantiene importantes limitaciones históricas y sociales. A ello podemos sumarle el hecho de que gran parte de la actividad científica en varias disciplinas no tiene objetivos estrictamente nomotéticos, por lo que sus pretensiones ecuménicas resultan injustificadas.

II. ¿Sólo son útiles para la invención de conjeturas los EC?

Como hemos mencionado, entre los psicoanalistas que difunden la necesidad de adoptar otras estrategias metodológicas, también se asume la suposición de que los EC son más útiles para generar hipótesis legaliformes, pero que conviene utilizar otros procedimientos para poder establecer la validez de las mismas. Es decir, el EC sólo puede ser útil en el contexto de descubrimiento pero no en el de justificación.

Una forma sencilla de mostrar porqué esta idea es equivocada es teniendo en cuenta que la falsación se produce a partir del hallazgo de ejemplos que contradicen las expectativas teóricas, por lo que algunos han sostenido incluso que los EC son valiosos fundamentalmente en los momentos en los que se decide poner a prueba una teoría (Eckstein, 1975/1992). Por ello, quizás el análisis en profundidad de casos sea la mejor manera de poner a prueba los modelos teóricos establecidos, en la medida en que es a partir de casos concretos que podemos dar con anomalías que se establezcan en falsaciones promotoras del cambio teórico.

Lo que es cada vez más evidente es que no hay razones serias para reducir el EC al contexto de descubrimiento. Kazdin, por ejemplo, diferencia un abanico de posibilidades de utilización del EC para hacer investigación, cuyos extremos estarían representados por un mínimo y un máximo control de las variables (Kazdin, 2001). Para muchos autores, este control máximo en los EC se logra sólo con diseños experimentales. Se trata de aquellos diseños en los que un solo individuo es su propio control y sirve para establecer una línea de base (o varias), una fase de intervención que manipula la o las variables independientes y una lectura de los efectos. Este tipo de diseño es posible bajo la suposición de que, "si un fenómeno es lo suficientemente básico, se tendrá que producir de forma similar en el resto de los individuos" (León y Montero, 1997, p. 227). A medida que nos alejamos de las posibilidades de control formal de las variables (sobre todo las independientes), el tipo de diseño pasa a ser encuadrado en otros formatos no experimentales (Roussos, 2007).

Cabe mencionar, por otro lado, que para algunos analistas, como Marshall Edelson, el diseño de EC no impide que se puedan utilizar cánones experimentales (como los propuestos por Grünbaum) para poner a prueba hipótesis psicoanalíticas utilizando el contexto clínico. Incluso sostiene que

En respuesta a los que exigen pruebas epidemiológicas y experimentales de la teoría psicoanalítica (p. ej., Grünbaurn 1984), he argumentado que el estudio de caso, ahora en descrédito, es sin embargo necesario para testear las hipótesis psiconíticas y, si está bien formulado, puede de hecho probar, no meramente generar, hipótesis ${ }^{27}$ (Edelson 1988, p. 278).

Coincido con Flyvbjerg en que "el estudio de casos es útil tanto para generar como para comprobar hipótesis, pero no se limita exclusivamente a estas actividades investigadoras” (2004 p. 44). En psicoanálisis esto es particularmente innegable, puesto que sólo puede accederse por esta vía al corazón de los fenómenos que resultan comúnmente meritorios de investigación, y, además de servir para inventar o poner a prueba 
conjeturas, resulta irremplazable, tanto para la transmisión e ilustración conceptual en la enseñanza, como para compartir el trabajo clínico ante la comunidad de pares.

\section{III. ¿Son los EC más propensos al sesgo de confirmación?}

Aunque pueda parecer añejada, la acusación de "subjetivo" es la más frecuentemente efectuada a los EC. No importa cuánto se haya subrayado, desde innumerables perspectivas, que el sujeto no puede sustraerse del campo cognitivo que investiga para obtener conocimiento científico. Difícilmente podamos eludir el hecho de que en ciencias de lo humano y, fundamentalmente en psicoanálisis, lo investigado son aspectos de la subjetividad a partir de la subjetividad, por lo que el conocimiento será afortunadamente subjetivo. Ello no implica, sin embargo, que el tipo de conocimiento obtenido con EC sea sesgado o arbitrario. No podría haber conocimiento empírico psicoanalítico que prescindiera del encuentro analítico, es decir, que no tome en cuenta los fenómenos que se producen de forma natural en un contexto que no se puede reproducir artificialmente mediante otros procedimientos.

La acusación más frecuente es la que afirma que el EC ofrecería mayor espacio al juicio arbitrario, por lo que sería más propenso a que el investigador incurra en sesgos cognitivos, como por ejemplo el sesgo de confirmación. Como señala Flyvbjerg, los investigadores experimentados en el uso de EC simplemente consideran esta crítica como muestra de una falta de conocimiento de lo que significa una investigación mediante esta metodología. Nada hay en el EC que impida controlar rigurosamente los sesgos cognitivos, que deben ser tenidos en cuenta como en cualquier otra metodología de investigación. Numerosos investigadores de diversas áreas señalan cómo sus expectativas (presupuestos, conceptos, prejuicios, hipótesis, etc.) han sido revisadas y modificadas durante la investigación con EC, mostrando cómo el material que esta metodología arroja permite sortear los sesgos. Además, como bien ha señalado Campbell (1975), si esa caricatura verificacionista fuera correcta, entonces la investigación con EC arrojaría innumerables explicaciones convincentes, provenientes de cada investigador, lo cual no sucede para nada en las investigaciones con EC.

Luego de revisar este prejuicio, Flyvbjerg concluye:

El estudio de casos no contiene un sesgo hacia la verificación de las nociones preconcebidas del investigador más marcado que otros métodos de investigación. Al contrario, la experiencia indica que el estudio de casos contiene un mayor sesgo hacia la falsación de nociones preconcebidas que hacia la verificación (Flyvbjerg, 2004, p. 52).

Aunque los anteriores son los principales presupuestos que suele asumir un sector de la comunidad psicoanalítica, para desestimar el EC como metodología de investigación válida y abogar por la promoción de otros caminos metodológicos, tales supuestos rara vez son puestos explícitamente en discusión. Si consideramos que la gran mayoría de los psicoanalistas que investigan continúan utilizando los EC para producir observables y evidencia para sus conjeturas, convendría que el diálogo reflexivo sobre estos aspectos metodológicos adquiera mayor lugar.

Por otro lado, los psicoanalistas defensores del EC también parecen asumir, quizás por la escasa reflexión metodológica sobre los aspectos considerados, algunas aseveraciones difíciles de aceptar. Quisiera enunciar dos de ellas y expedirme críticamente sobre las mismas:

a) El método clínico tradicional es el único método de investigación posible en psicoanálisis. Incorporar otros procedimientos metodológicos atenta contra la pureza del psicoanálisis.

Respecto de la primera, quisiera subrayar una suerte de paradoja que ha pasado inadvertida. No han faltado psicoanalistas que describieran con el mote de "positivistas" 28 a todos aquellos que pretendieron indagar su mismo campo de fenómenos con métodos diversos al EC psicoanalítico. Paradójicamente, son ellos los que hacen revivir lo más rancio del espíritu positivista: janteponen el método al objeto! A mi modo de ver, el método psicoanalítico tradicional sigue siendo la principal herramienta metodológica para la investigación psicoanalítica, pero eso no quiere decir que sea la únicaposible, ni, mucho menos, que con ella baste para obtener todas las respuestas que necesitamos a las preguntas que se nos presentan ${ }^{29}$. Lo que comúnmente suele 
olvidarse, generalmente por parte de los defensores de la exclusividad de la investigación clínica, es que el tipo de procedimientos a utilizar debe ser solidario del tipo de problemas que merecen nuestra atención. Pretender que el procedimiento sea un a priori metodológico es reducir obstinadamente el abanico de posibilidades. Es autocercenar tanto el campo de formulación de los problemas como el de las respuestas posibles.

La segunda afirmación, en sintonía con la primera, es una aseveración que difícilmente pase el plano ideológico. No sólo que es cada vez más difícil establecer criterios comúnmente aceptados para poder identificar qué entender y qué no por psicoanálisis, sino que, además, la idea de una "pureza"o "esencia atemporal" psicoanalítica contraría la dinámica evolutiva de las disciplinas. Me refiero a que si se observa el devenir histórico de un dominio cognitivo, al menos en las ciencias empíricas, se percibe con facilidad que las fronteras son móviles, y que a menudo hay solapamientos considerables, convergencias, diluciones, etc. En virtud de ello, no parece razonable suponer que lo que hoy conocemos como psicoanálisis (incluso si pudiésemos definirlo con aceptable univocidad) seguirá siendo considerado como tal dentro de cincuenta o cien años, por ejemplo. Asumir una identidad en constante transformación quizás resulte más productivo y saludable -al menos para el asunto al que me estoy refiriendo- que presuponer una identidad en términos esencialistas.

\section{Conclusiones}

Al considerar algunas ${ }^{30}$ tipologías inherentes a la investigación psicoanalítica, en primer lugar hemos visto por qué la apelación a ciertas críticas epistemológicas y metodológicas ha sido un factor fundamental para justificar el desarrollo de investigación extraclínica en el interior de dicho campo. Se mostró el modo en que este tipo de investigaciones suele derivarse de alguna demanda incumplida de legitimidad epistémica, basada en las supuestas deficiencias del procedimiento tradicional de investigación psicoanalítica (el EC) y en una concepción del conocimiento científico que mantiene por ideal a las ciencias naturales experimentales. Éste pareciera haber sido un punto de partida comúnmente asumido por aquellas orientaciones psicoanalíticas que han venido promocionando tanto los estudios sobre eficacia terapéutica como el desarrollo interdisciplinario con perspectivas neurocientíficas, a los fines de reducir el aislamiento disciplinar y de recuperar legitimidad epistémica. Por el contrario, quienes más se han venido resistiendo a la investigación empírica son aquellos analistas que no comulgan con esa referencia naturalista para construir conocimientos psicoanalíticos, y que defienden, aunque no siempre de manera suficientemente fundamentada, el EC como metodología necesaria y suficiente para tal finalidad.

$\mathrm{Al}$ considerar el problema del estatuto del EC desde un punto de vista metateórico, advertimos que su desestimación se sostiene en algunos presupuestos que ameritan ser revisados y discutidos. Se explicitaron tres de las principales objeciones que suele recibir dicha herramienta en el campo general de las ciencias de lo humano (objeciones reencontrables en el interior del psicoanálisis) y se analizaron los principales argumentos metodológicos y epistemológicos sobre los que tales asertos se apoyan. Lo examinado muestra que no hay razones para asumir irrestrictamente ese conjunto de afirmaciones y que, por el contrario, es posible defender la legitimidad epistémica de los EC para la investigación en psicoanálisis. Esto no significa que dicha estrategia sea la única posible ni que prescindir de ella atente contra la supuesta pureza del psicoanálisis: si bien la indagación clínica pareciera ser condición necesaria para la gran mayoría de los problemas psicoanalíticos, no siempre es una condición suficiente. Debería quedar claro, entonces, que no hay razones epistémicas para pretender defender la exclusividad del EC como metodología de investigación psicoanalítica y que las estrategias metodológicas deben ser secundarias en relación a los problemas por abordar.

Poder discutir estos y otros problemas metateóricos constituye, ante la diversidad de propuestas de investigación que hoy en día cohabitan nuestro campo disciplinar, una labor cada vez más necesaria para abogar por un crecimiento racional del psicoanálisis en el siglo XXI. 


\section{Referencias bibliográficAs}

Aguillaume, R. (2016). Epistemology and psychoanalysis: One psychoanalysis or two? International Forum of Psychoanalysis, 25(3), 149.

Archenti, N. (2007). Estudio de casos/s. En A. Marradi., N. Archenti y J.I. Piovani, Metodología de las Ciencias Sociales (pp. 237-246). Buenos Aires: Emecé.

Ávila-Espada, A., y Mitjavila, M. (2003). El método del plan de acción latente del terapeuta (TLAP). Un nuevo método para predecir la contribución cualitativa del terapeuta al resultado de tratamiento. Subjetividady Procesos Cognitivos, 3, 9-36.

Ávila Espada, A., y García de la Hoz, A. (2014). Historia, núcleo conceptual y controversias epistemológicas del modelo psicodinámico. En A. Ávila Espada, B. Rojí Menchaca y L. Á. Saúl Gutiérrez (Coord.), Introducción a los tratamientos psicodinámicos (pp. 63-98). Madrid: UNED.

Bernardi, R. (2015). Investigación clínica e investigación empírica sistemática: ¿qué nos enseñan las controversias actuales? International Journal of Psycho-Analysis en Español, 1(3), 836-866.

Bleichmar, N., y Leiberman, C. (1997). Problemas epistemológicos en la teoría psicoanalítica. En El psicoanálisis después de Freud: teoría y clínica (pp. 477-496). Buenos Aires: Paidós.

Bornstein, R. (2001). The impending death of psychoanalysis. Psychoanalytic Psychology, 18, 3-20.

Bornstein, R., y Masling, J. (eds.) (1998a). Empirical Perspectives on the Psychoanalytic Unconscious. Washington DC: American Psychological Association.

Bornstein, R. \& Masling, J. (eds.) (1998b). Empirical studies of the therapeutic hour. Washington DC: American Psychological Association.

Bucci, W. (1997). Psychoanalysis and Cognitive Science: a Multiple Code Theory. Nueva York: Guilford Press.

Buzzoni, M. (2001). The Operationalistic and Hermeneutic Status of Psychoanalysis. Journal for General Philosophy of Science, 32, 131-165.

Campbell, D. T. (1975). Degrees of Freedom and the Case Study. Comparative Political Studies, 8(1), 178-191.

Dahl, H. (1998). The Voyage of el Rubaiyat to the Discovery of FRAMES. En R. F. Bornstein \& J. M. Masling (Eds.), Empirical Studies of the Therapeutic Hour (pp. 179-227). Washington DC: American Psychological Association.

Dahl, H. (1988). Frames of mind. En H. Dahl, H. Kächele, \& H. Thomä (eds.), Psychoanalytic Process Research Strategies (pp. 51-66). Berlin: Springer-Verlag.

Dreher, A. (2000). Foundations for Conceptual Research. London, Karnac Books.

Dreher, A. (2005). Conceptual research. En E.S. Person, A.M. Cooper \& G.O. Gabbard (eds), Textbook of Psychoanalysis (pp. 361-375). Arlington, VA: American Psychiatric Publishing, Inc.

Eagle, M., \& Wolitzky, D. (2011). Systematic empirical research versus clinical case studies: a valid antagonism? Journal of the American Psychoanalytic Association, 59(4), 791-818.

Eckstein, H. (1975/1992). Case Studies and Theory in Political Science. En Regarding Politics: Essays on Political Theory, Stability, and Change (pp. 118-164). Berkeley: University of California Press.

Edelson, M. (1988). Psychoanalysis: A Theory in Crisis. Chicago: University of Chicago Press.

Erwin, E. (2015). Psychoanalysis and philosophy of science: basic evidence. En S. Boag, L. Brakel \& V. Talvitie (Eds.), Philosophy, Science, and Psychoanalysis. A critical meeting, (pp. 37-58). London: Karnac.

Fisher, S. \& Greenberg, R. (1996). Freud scientifically reappraised: Testing the theories and therapy. New York: Wiley $\&$ Sons.

Flyvbjerg, B. (2004). Cinco malentendidos acerca de la investigación mediante los estudios de caso. Revista Española de Investigación Sociológica, 106, 33-62. Recuperado de http://www.reis.cis.es/REIS /PDF/REIS _106_041167998142322.pdf.

Fonagy, P. (1982). The integration of psychoanalysis and experimental science: a review. International Review of PsychoAnalysis, 9, 125-145. 
Fonagy, P. (1999). Reflections on psychoanalytic research problems - an anglosaxon view. En An Open door review of outcome studies in psychoanalysis (pp. 10-27). Londres, IPA Report.

Fonagy, P. (2000). Grasping the nettle: Or why psychoanalytic research is such an irritant. The British Psycho-Analytical Society, 36, 28-36.

Fonagy, P. (2003). Genética, psicopatología evolutiva y teoría psicoanalítica: el argumento para terminar con nuestro (no tan) espléndido aislamiento. Aperturas psicoanalíticas: Revista de psicoanálisis, 2(15).

Fonagy, P. (2015). La eficacia de las psicoterapias psicodinámicas: una actualización. World Psychiatry (en español), $14,137-150$.

Freud, S. (1912/2004). Consejos al médico sobre el tratamiento psicoanalítico. En Obras Completas, tomo XII [¿págs.?]. Buenos Aires: Amorrortu.

Freud, S. (1933/2004). Nuevas conferencias de introducción al psicoanálisis. $34^{\circ}$ conferencia. Esclarecimientos, aplicaciones, orientaciones. En Obras Completas, tomo XXII [¿págs.?]. Buenos Aires: Amorrortu.

Fusella, P. (2014). Hermeneutics versus science in psychoanalysis: a resolution to the controversy over the scientific status of psychoanalysis. Psychoanalytic review, 101(6), 871-894.

Gaeta, R. (2012). El fantasma del positivismo en las ciencias sociales. Filosofía Unisinos, 13(2-suppl.), 225-249.

Green, A. (1996). What kind of research for psychoanalysis? International Psychoanalysis: Newsletter of the International Psychoanalytical Association 5, 10-14.

Green, A. (2000). Science and science fiction in infant research. En J. Sandler, A. M. Sandler \& R. Davies (eds.). Clinical and observational psychoanalytic research: Roots of a controversy. André Green \& Daniel Stern (pp. 41-72). London: Karnac.

Green, A. (2005/2006). La ilusión del terreno común y el pluralismo mítico. Psicoanálisis, 28(3), 657-665.

Grünbaum, A. (1993). Validation in the clinical theory of psychoanalysis: A study in the philosophy of psychoanalysis. Madison: International Universities Press.

Grünbaum, A. (1984). The Foundations of Psychoanalysis: A Philosophical Critique. California: University of California Press.

Grünbaum, A. (1988). The Role of the Case Study Method in the Foundations of Psychoanalysis. Canadian Journal of Philosophy, 18(4), 623-658.

Gunderson, J. G., \& Gabbard, G. O. (1998). Making the case for psychoanalytic therapies in the current psychiatric environment. Journal of the American Psychoanalytic Association, 47, 679-704.

Hofmann, S. G. (2016). Psychodynamic therapy: a poorly defined concept with questionable evidence. Evidence Based Mental Health, 19, 63.

Hoffman, I. Z. (2009). Doublethinking our way to "scientific" legitimacy: The desiccation of human experience. Journal of the American Psychoanalytic Association, 57(5), 1043-1069.

Jiménez, J. P. (2004). Validez y validación del método psicoanalítico (alegato sobre la necesidad del pluralismo metodológico y pragmático en psicoanálisis). Revista Aperturas Psicoanalíticas, 18.

Jones, E. E. (2000). Therapeutic Action: A Guide to Psychoanalytic Therapy. New Jersey: Jason Aronson.

Kächele, H. (1992). Investigación psicoanalítica: 1930-1990. Revista Chilena de Psicoanálisis, 9(1), 55-68.

Kächele, H.; Schachter, J. \& Thomä, H. (2011). From psychoanalytic narrative to empirical single case research: Implications for psychoanalytic practice. New York: Routledge.

Kandel, E. R. (1998) A new intellectual framework for psychiatry. American Journal of Psychiatry, 155, 457-469.

Kandel, E. R. (1999). Biology and the future of psychoanalysis: A new intellectual framework for psychiatry revisited. American Journal of Psychiatry, 156, 505-524.

Kaplan-Solms, K. \& Solms, M. (2000/2005). Estudios clinicos en neuropsicoanálisis: introducción a la neuropsicología profunda. Colombia: Fondo de Cultura Económica.

Kazdin, A. E. (2001). Métodos de investigación en psicología clinica (3ª.ed.). México: Pearson Educación. 
Kernberg, O. (2006). Research anxiety: A reply to Roger Perron's comments, International Journal of Psychoanalysis, $8,933-937$.

Kline, P. (1972/2014). Fact and fantasy in freudian theory. New York: Routledge.

Lancelle, G. (1997). El psicoanálisis y la investigación en escorzo desde una perspectiva clínica. Investigación en Psicoanálisis. Revista de la Asociación Psicoanalítica de Buenos Aires, 19(1-2), 119-156.

Leichsenring, F.; Abbass, A.; Gottdiener, W.; Hilsenroth, M.; Keefe, J. R.; Luyten, P.; Rabung, S. \& Steinert, C. (2016). Psychodynamic therapy: a well-defined concept with increasing evidence. Evidence Based Mental Health, 19(2), 64.

Leichsenring, F.; Luyten, P.; Hilsenroth, M. J.; Abbass, A.; Barber, J. P.; Keefe, J. R.; Leweke, F.; Rabung, S. \& Steinert, C. (2015). Psychodynamic therapy meets evidence-based medicine: a systematic review using updated criteria. The Lancet Psychiatry, 2(7), 648-660.

Lemlij, M. (2013). La investigación en la API: Reflexiones de un ex tesorero. Calibán: Revista Latinoamericana de Psicoanálisis, 11(2), 150-152.

León, O., y Montero, I. (1997). Diseño de investigaciones. Introducción a la lógica de investigación en Psicología y Educación. España: Mc Graw-Hill.

Leuzinger-Bohleber, M. \& Kächele, H. (eds.) (2015). An open door review of outcome and process studies in psychoanalysis. Third Edition. Londres: International Psychoanalytical Association.

Leuzinger-Bohleber, M. (2015). Psychoanalysis as a "science of the unconscious" and its dialogue with the neurosciences and embodied cognitive science: some historical and epistemological remarks. En Finding the Body in the Mind: Psychoanalysis, Neurosciences, Embodied Cognitive Science in Dialogue (pp. 1-18). London: Karnac.

Leuzinger-Bohleber, M., \& Bürgin, D. (2003). Pluralism and unity in psychoanalytic research: Some introductory remarks. En M. Leuzinger-Bohleber, A.U. Dreher, \& J. Canestri (eds), Pluralism and Unity? Methods of Research in Psychoanalysis (pp. 1-25).London: International Psychoanalytical Association.

Liberman, D. (1970). Lingüistica, interacción comunicativa y proceso psicoanalitico. Buenos Aires: Nueva Visión.

Lothane, Z. (1998). El eterno Freud: el método frente al mito y la malicia de los detractores de Freud. Revista de la Asociación Española de Neuropsiquiatría, 18(66), 269-292.

Luborsky, L. (1976). Helping alliances in psychotherapy: The groundwork for a study of their relationship to its outcome. En J. L. Claghorn (Ed.), Successful psychotherapy (pp. 92-116). New York: Brunner / Mazel.

Luborsky, L. (1977). Measuring a pervasive psychic structure in psychotherapy: The Core Conflictual Relationship Theme. En N. Freedman \& S. Grand (Eds.), Communicative structures and psychic structures (pp. 367-395). New York: Plenum.

Luborsky, L. \& Crits-Christoph, P. (1990). Understanding Transference: The Core Conflictual Relationship Theme Method. Nueva York: Basic Books.

Luyten, P., Blatt, S. J., y Corveleyn, J. (2006). Salvando la distancia entre el positivismo y la hermenéutica en la investigación psicoanalítica. Aperturas psicoanalíticas. Revista de psicoanálisis, 24, 571-610.

Maldavsky, D., y Liberman, D. (2004). La investigación psicoanalitica del lenguaje: algoritmo David Liberman. Buenos Aires: Lugar.

Masling, J. M. \& Bornstein, R. F. (eds.) (1996). Psychoanalytic Perspectives on Developmental Psychology. Washington: American Psychological Association.

Moser, U. (1989/1992). On-Line and Off-Line, Practice and Research: A Balance. En M. Leuzinger-Bohleber, H. Schneider, \& R. Pfeifer (eds.), Two Butterflies on My Head... Psychoanalysis in the Interdisciplinary Scientific Dialogue (pp. 181-196). New York: Springer. Originalmente publicado en Psyche - Zeitschrift für Psychoanalyse, $45,315-34$.

Nasio, J. D. (2001). ¿Qué es un caso? En J. D. Nasio (ed.), Los más famosos casos de psicosis (pp. 15-37). Buenos Aires: Paidós.

Pérez Serrano, G. (1994). Investigación cualitativa. Retos e interrogantes. Madrid: La Muralla. 
Perron, R. (1999). Reflections on psychoanalytic research problems - the French -speaking view. En An Open door review of outcome studies in psychoanalysis (pp. 3-9). Londres: IPA Report.

Roussos, A. (2007). El diseño de caso único en investigación en psicología clínica. Revista Argentina de Clínica Psicológica, XVI, 3, 261-270.

Rubistein, A., y Trotta, L. (2004). La eficacia del psicoanálisis: importancia y estado actual del tema. En Memorias de las XI Jornadas de Investigación, 1-12. Buenos Aires: Facultad de Psicología de la Universidad de Buenos Aires.

Safran, J. D. (2001). When worlds collide: Psychoanalysis and the empirically supported treatment movement. Psychoanalytic dialogues, 11(4), 659-681

Safran, J. D. (2010). Discussion by Jeremy D. Safran. Panel discussion on "Clinical and Empirical Issues: Disagreements and Agreements". Paper presented at the Annual Meeting of the Rapaport-Klein Study Group, Austen Riggs Center.

Sarnoff, I. (1971). Testing Freudian concepts: An experimental social approach. New York: Springer.

Schafer, R. (1976). A New Language for Psychoanalysis. New Haven: Yale University Press.

Shapiro, T. \& Emde, R. (1995). Research in Psychoanalysis: process, development, outcome. Madison, CT: International Universities Press.

Shedler, J. (2002). A new language for psychoanalytic diagnosis. Journal of American Psychoanalytic Association, 50(2), 429-456.

Shedler, J. (2010). The efficacy of psychodynamic psychotherapy. The American psychologist, 65(2), 98.

Shevrin, H., \& Fritzler, D. E. (1968). Visual evoked response correlates of unconscious mental processes. Science, 161(3838), 295-298.

Shevrin, H.; Snodgrass, M.; Brakel, L. A.; Kushwaha, R.; Kalaida, N. L.; \& Bazan, A. (2013). Subliminal unconscious conflict alpha power inhibits supraliminal conscious symptom experience. Frontiers in Human Neuroscience, 7 , 544 .

Soler, C. (1988/2007). Finales de análisis. Buenos Aires: Manantial.

Solms, M. (2001). The interpretation of dreams and the neurosciences. Psychoanalysis and History, 3, 79-91.

Solms, M. (2013). The Conscious Id. Neuropsychoanalysis, 15, 5-19.

Solms, M., \& Turnbull, O. H. (2011). What is neuropsychoanalysis?. Neuropsychoanalysis, 13(2), 133-145.

Spence, D. P. (1984). Narrative truth and historical truth: Meaning and interpretation in psychoanalysis. New York: WW Norton \& Company.

Stake, R. E. (1999). Investigación con Estudio de casos. Madrid: Morata.

Stake, R. E. (2013). Estudios de casos cualitativos. En N. Denzin y Y. Lincoln (eds), Estrategias de investigación cualitativa (Vol. III) (pp. 154-197). Barcelona: Gedisa.

Stern, D. N. (2000). The relevance of empirical infant research to psychoanalytic theory and practice. En J. Sandler, A.- M. Sandler \& R. Davies (eds.), Clinical and observational psychoanalytic research: Roots of a controversy. André Green \& Daniel Stern (pp. 73-90). London: Karnac.

Strenger, C. (1991). Between hermeneutics and science: An essay on the epistemology of psychoanalysis. Madison: International Universities Press.

Simons, H. (2011). El estudio de caso: Teoria y Práctica. Madrid: Ediciones Morata.

Thomä. H., y Kächele. H. (1985/1989). Teoria y práctica del psicoanálisis. I fundamentos. Barcelona: Herder.

Thomä, H., y Kächele, H. (1999/2000). Memorándum sobre la reforma de la formación psicoanalítica. Intersubjetivo, 2(1), 101-114. Originalmente publicado en: IPA Newsletters, 8, 33-35.

Wallerstein, R. S. \& Sampson, H. (1971). Issues in research in the psychoanalytic process. International Journal of Psychoanalysis, 52, 11-50.

Wallerstein, R. S. (1993). Investigación psicoanalítica. Informativo de la API, Psicoanálisis Internacional, verano de 1993. 
Wallerstein, R. S. (2001). The generations of psychotherapy research: An overview. Psychoanalytic Psychology, 18(2), 243-267.

Wallerstein, R. S. (2006). Psychoanalytic therapy research: Its history, its present status, and its projected future. En PDM Task Force. Psychodynamic diagnostic manual (pp.511-535). Silver Spring, MD: Alliance of Psychoanalytic Organizations.

Wallerstein, R. S. (2005/2006). ¿Será el pluralismo psicoanalítico un estado duradero de nuestra disciplina? Psicoanálisis, 28(3), 649-655. (Original publicado en: International Journal of Psychoanalysis, 86, 623-626)

Westen, D. (1998). The scientific legacy of Sigmund Freud: Toward a psychodynamically informed psychological science. Psychological Bulletin, 124, 333-371.

Westen, D. (1999). The scientific status of unconscious processes: is Freud really dead? Journal of the American Psychoanalytic Association, 47(4), 1061-1106.

Wolff, P. H. (1996). The irrelevance of infant observations for psychoanalysis. Journal of the American Psychoanalytic Association, 44, 369-392.

Yin, R. K. (2002). Case study research: Design and methods. Newbury Park: Sage Publications. Recuperado de http: //www.pdffactory.comhttp://www.polipub.org/documentos/YIN\%20ROBERT\%20.pdf.

Ynoub, R. (2015). Cuestión de método. Aportes para una metodología crítica. Tomo I. México: Cengage Leing.

Yovell, Y., Solms, M. \& Fotopoulou, A. (2015). Argumento a favor del neuropsicoanálisis: por qué el diálogo con la neurociencia es necesario pero no suficiente para el psicoanálisis. International Journal of Psycho-Analysis en Español, 1, 1782-1833.

Znaniecki, F. (1934). The Method of sociology. New York: Rinehart \& Company.

Zukerfeld, R. (2001). Alianza terapéutica y encuadre analítico. VERTEX Revista Argentina de Psiquiatría, 12, 211-20.

\section{Notas}

1. The psychoanalytic therapist and practitioner is (...) an on-line scientist (...) This is the case for two reasons. First, therapies as a part of the system create the system they observe and change it continuously, and second, therapists must be "scientifically" active during the therapy situation. They produce knowledge. The validity of one's own action in this area is left to intuitive certainty, a phenomenon - as far as I know - to which psychotherapy research has not yet turned its attention".

2. Existe cierto consenso general sobre la evolución histórica del campo psicoanalítico: a pesar de la pluralidad de orientaciones teóricas y su desarrollo interno, cabe la posibilidad de reencontrar al interior del psicoanálisis una oposición entre tradiciones naturalistas y tradiciones hermenéuticas (Strenger, 1991; Bleichmar y Leiberman, 1997; Buzzoni, 2001; Luyten et al., 2006; Fusella, 2014; Ávila Espada y García de la Hoz, 2014; Erwin, 2015; Aguillaume, 2016). Aunque no desconozco sus limitaciones, aquí voy a adherir a esa diferenciación, entre otras cosas porque permite contextualizar mejor los debates metodológicos de los que voy a ocuparme.

3. "...if psychoanalysis is to have a future as a scientific enterprise, it very probably does not lie with the clinical case history method, but with other testing designs."

4. "There are three conditions that should be met for clinical research to be an adequate sole methodology of psychoanalytic theory building. These are: (a) a close logical tie between theory and practice, (b) appropriate deductive reasoning in relation to clinical material and (c) the unambiguous use of terms".

5. "The case study by itself, however, is insufficient as a method of investigation. It needs to be supplemented by other confirmatory procedures such as replication, detailed experimental studies, anatomical, genetic and neurophysiological investigations".

6. Grabaciones de audio y video resultan las más frecuentes. Para una reseña y análisis crítico de las ventajas y desventajas de estos procedimientos, cf. "The Pro and Contra of Tape Recordings" (Kächele et al., 2011, p. 12) y Wallerstein \& Sampson (1971).

7. "The future of psychoanalysis, if it is to have a future, is in the context of an empirical psychology, abetted by imaging techniques, neuroanatomical methods, and human genetics. Embedded in the sciences of human cognition, the ideas of psychoanalysis can be tested, and it is here that these ideas can have their greatest impact".

8. "psychoanalysis still represents the most coherent and intellectually satisfying view of the mind". 
9. “....as a research tool this particular method has exhausted much of its novel investigative power. One hundred years after its introduction (...) We must, at last, acknowledge that (...) is not a sufficient basis for a science of mind"

10. "we do now have neuroscientific methods that enable us to study the dynamic nature of the mind and to identify the neural organization of its unconscious substructure (...) Freud would, in our opinion, have considered this a welcome and wholly legitimate development of the work that he pioneered".

11. "...neuroscience offers a second perspective on the unknowable 'thing' that we call the mental apparatus, the thing that Freud attempted to describe for the first time in his metapsychology".

12. Así presentan la perspectiva de sus investigaciones estos autores: "Irónicamente, debemos el desarrollo de un procedimiento clínico para analizar la estructura profunda de la vida mental subjetiva al hecho de que Freud abandonó los métodos neurocientíficos de investigación, cuando se dio cuenta de que estos eran incapaces de dar cabida a la naturaleza dinámica y 'virtual' del proceso mental. Ahora nos ha llegado la hora de reintroducir los frutos de los trabajos de Freud en el campo neurocientífico del cual surgieron originalmente (...) Lo que recomendamos, por lo tanto, y lo que creemos que ha de proveer los fundamentos para la integración duradera del psicoanálisis y la neurociencia, es una investigación totalmente psicoanalítica de los pacientes con lesiones neurológicas focales. En otras palabras, recomendamos que proyectemos la organización neurológica de los procesos mentales humanos que el psicoanálisis ha revelado, usando una versión modificada del método de Luria del análisis del síndrome, para estudiar la estructura profunda de los cambios mentales en pacientes neurológicos de manera que dicha estructura pueda discernirse dentro de un marco psicoanalítico" (Kaplan-Solms \& Solms, 2000/2005, p. 66).

13. "In response to these challenges, we applied our research method in order to find independent evidence for the validity of the constructs of repression and unconscious conflict".

14. "Neuroscience is no more the final court of appeal for psychoanalysis than psychoanalysis is for neuroscience. The final court of appeal for psychoanalysts is the clinical situation".

15. "...evidence that patients who receive psychodynamic therapy not only maintain therapeutic gains but continue to improve over time".

16. "Nonpsychodynamic therapies may be effective in part because the more skilled practitioners utilize interventions that have long been central to psychodynamic theory and practice".

17. "In writing this article, I could not help being struck by a number of ironies. One is that academicians who dismiss psychodynamic approaches, sometimes in vehement tones, often do so in the name of science. Some advocate a science of psychology grounded exclusively in the experimental method. Yet the same experimental method yields findings that support both psychodynamic concepts".

18. "Just as antipsychoanalytic sentiment may have impeded dissemination of this research in academic circles, distrust of academic research methods may have impeded dissemination in psychoanalytic circles".

19. Recordemos, de pasada, que éste es el problema que Freud supo advertir en los estudios estadísticos sobre la eficacia. Es clara su manifestación luego de que el Instituto Psicoanalítico de Berlín hubiera publicado en 1930 el primer estudio estadístico, que abarcó diez años de labor: "los éxitos terapéuticos no justifican la jactancia, pero tampoco dan lugar a avergonzarse. Sin embargo, tales estadísticas no esclarecen nada; el material procesado es tan heterogéneo que sólo muy grandes números significarían algo. Lo mejor es indagar las propias experiencias” (Freud, 1933/2004, p. 141).

20. Otra revisión reciente con similares resultados es la de Leichsenring \& Klein (2015). Para una lectura interesante de algunas dificultades en el establecimiento de criterios para efectuar este tipo de metanálisis, cf. Leichsenring et al. (2015). Un debate reciente sobre estos aspectos puede encontrarse en las objeciones de "poca claridad" de Stefan Hofmann (2016) a los metanálisis existentes y en la réplica de Leichsenring et al. (2016).

21. "Grasping the nettle: Or why psychoanalytic research is such an irritant"

22. "They see no way to quantify important psychoanalytic constructs without trivializing and distorting them. They are turned off by the kind of reductionism that has characterized empirical research in the past. From the observation 'it has not been done' they have reasoned 'it cannot be done'".

23. "We have competing models with different implications for technique, and advocates of each model regularly publish case studies "demonstrating" the superiority of their own approach. The case studies do not seem to change anyone's mind, because analysts of different persuasions see different things in much the same case material".

24. "However much we may value the uniqueness of our patients, we do not approach them in a vacuum. If we have expertise in treating human suffering, it is precisely because we can recognize commonalities across patients and understand their suffering in the broader context of familiar patterns and syndromes".

25. Esta distinción de Yin nos recuerda la diferenciación temprana del sociólogo polaco Florian Znaniecki entre inducción enumerativa e inducción analítica. Esta última consiste en una examinación secuencial de los datos en busca de categorías de fenómenos, que parte de hipótesis de trabajo obtenidas en los casos iniciales que van siendo permanentemente ajustadas conforme se conocen más casos (Znaniecki, 1934). Coincido con Roxana Ynoub en que este proceso no es enteramente inductivo y que lo esencial se comprende mejor bajo la lógica de las inferencias abductivas (Ynoub, 2015). 
26. “...el caso crítico se puede definir como un caso con importancia estratégica para el problema en general. Por ejemplo, una clínica de medicina ocupacional quiere investigar si las personas que trabajaban con disolventes orgánicos padecen daños cerebrales. En lugar de elegir una muestra representativa de entre las empresas del sector que usan disolventes orgánicos, la clínica localiza estratégicamente un lugar de trabajo que cumple todas las medidas de seguridad, higiene, calidad del aire, etc. Esta empresa modelo se convierte en el caso crítico: si en esas instalaciones la clínica encuentra el tipo de secuelas cerebrales relacionado con los disolventes orgánicos, entonces es probable que ese problema se dé también en otras empresas que observan menos las medidas de seguridad e higiene. Con este tipo de elección estratégica se puede ahorrar tiempo y dinero en la investigación de un determinado problema” (Flyvbjerg, 2004, p. 45).

27. "In response to those who demand epidemiologic and experimental tests of psychoanalytic theory (e.g., Grünbaum, 1984), I have argued that the case study, now held in disrepute, is nevertheless necessary to test psychoanalytic hypotheses and, if properly formulated, can indeed test, not merely generate, hypotheses".

28. En las ciencias de lo humano se suele otorgar el mote de "positivistas" a un vasto y difuso conjunto de perspectivas, con la intensión de significar así su descalificación; al punto que cabe hablar de una suerte de "fantasma del positivismo en las Ciencias Sociales" (Gaeta, 2012).

29. En este escrito no voy a abordar un problema que aparece involucrado en estas consideraciones: el de la unión inseparable entre curar e investigar. En virtud de su densidad, reservo su tratamiento para un trabajo futuro.

30. Deseo dejar en claro, en virtud de las observaciones de uno de los evaluadores del artículo, que no he pretendido exhaustividad alguna, sino que me he limitado a reseñar las tipologías que considero más relevantes respecto de las formas de investigar en psicoanálisis. Seguramente, en función de otros objetivos epistémicos distintos, serían posibles o necesarios otros ordenamientos de dicho campo. 